\title{
Multifocal spectral-domain optical coherence tomography based on Bessel beam for extended imaging depth
}

Luying Yi

Liqun Sun

Wuwen Ding 


\title{
Multifocal spectral-domain optical coherence tomography based on Bessel beam for extended imaging depth
}

\author{
Luying Yi, Liqun Sun, ${ }^{*}$ and Wuwen Ding \\ Tsinghua University, State Key Laboratory of Precision Measurement Technology and Instruments, Department of Precision Instruments, \\ Beijing, China
}

\begin{abstract}
To advance the practical application of optical coherence tomography (OCT) in the field of biomedical imaging, the imaging depth must be extended without sacrificing resolution while maintaining sufficient sensitivity. However, there is an inherent trade-off between lateral resolution and depth of field (DOF) in OCT. To address this shortcoming, this article proposes a multifocal Bessel beam spectral-domain optical coherence tomography (MBSDOCT) capable of increasing the DOF with unchanged lateral resolution and a high signal-tonoise ratio. The proposed technique is demonstrated by simulation and experiment. A three-focal MBSDOCT with an axicon lens theoretically achieved a DOF of $\sim 6 \mathrm{~mm}$ with a lateral resolution of $\sim 13 \mu \mathrm{m}$. In imaging experiments performed on the acinar cells of orange tissue, a measured DOF of $\sim 4 \mathrm{~mm}$ was demonstrated with a sensitivity penalty of $\sim 18.1 \mathrm{~dB}$, relative to the Gaussian beam spectral-domain OCT, with a 9-mW light source. (c) 2017 Society of Photo-Optical Instrumentation Engineers (SPIE) [DOI: 10.1117/1.JBO.22.10.106016]
\end{abstract}

Keywords: optical coherence tomography; Bessel beam; multifocal image; imaging depth.

Paper 170466RR received Jul. 14, 2017; accepted for publication Oct. 11, 2017; published online Oct. 27, 2017.

\section{Introduction}

Optical coherence tomography (OCT), a noninvasive threedimensional (3-D) imaging modality based on low coherence interferometry, ${ }^{1,2}$ has demonstrated effectiveness for in vivo imaging, ${ }^{3-5}$ and in particular for biomedical applications. ${ }^{6-9}$ To advance OCT technology for practical applications, the imaging depth must be extended without sacrificing resolution or sensitivity. Lenses with low numerical aperture are usually used in conventional Gaussian beam OCT to maintain a long depth of field (DOF); nevertheless, this approach incurs low lateral resolution. Different approaches have been taken to overcome this inherent trade-off between lateral resolution and DOF in OCT; those approaches can be classified into two categories in terms of the focusing beam: Gaussian beam OCT (GOCT) and Bessel beam OCT (BOCT).

There are two kinds of GOCT with extended DOF: multifocal GOCT (MGOCT) $)^{10,11}$ and interferometric synthetic aperture microscopy (ISAM). ${ }^{12-16}$ In MGOCT, multiple apertures are used simultaneously in the scanning process. Each aperture is focused at a different depth during the scan. Compared with each set of OCT data, the MGOCT shows an improved resolution over the whole depth; however, the DOF of a Gaussian beam with high resolution is very small. In contrast, ISAM takes the OCT data and corrects for the spreading of the beam by applying a specific algorithm, resulting in an infinite extension of the DOF and invariant spatial resolution along the depth axis. However, in ISAM, the effective DOF is limited because the signal-to-noise ratio (SNR) decreases with $1 / z$, where $z$ is the depth coordinate. $\mathrm{Xu}$ et al. ${ }^{17}$ proposed the multifocal ISAM (MISAM), which can further extend the DOF.

*Address all correspondence to: Liqun Sun, E-mail: sunlq@mail.tsinghua.edu .cn
BOCT $^{18,19}$ extends the DOF using a Bessel beam to illuminate the sample. The Bessel beam is generated using an axicon lens. The scattered signal is collected using either the axicon lens or a conventional objective lens. Lee and Rolland ${ }^{19}$ reported a doublepass BOCT that achieved a measured invariant resolution of $\sim 8 \mu \mathrm{m}$ across a 4-mm DOF measured using the microoptic axicon while achieving an invariant sensitivity of $\sim 80 \mathrm{~dB}$ with a $25-\mathrm{mW}$ input power. Leitgeb et al. ${ }^{20}$ proposed a nondual path imaging scheme to image biological samples and reported a $1.5-\mu \mathrm{m}$ resolution across a $200-\mu \mathrm{m}$ DOF. Curatolo et al. ${ }^{21}$ reported that superior contrast (by up to $\sim 40 \%$ ) may be obtained over an extended depth range by MGOCT compared with BOCT. However, the GOCT has a very small DOF when the lateral resolution is high. Even with the MGOCT, it is difficult to achieve the same DOF as is achieved by the BOCT for a given lateral resolution when the SNR penalty of the BOCT can be accepted.

Recent developments in detector technology have enabled high acquisition speeds; therefore, spectral-domain optical coherence tomography (SDOCT), which is capable of high-resolution, real-time, and 3-D imaging of the internal structures of biological tissues, is currently an important tool for biological tissue imaging. ${ }^{22-26}$ To address the trade-off between DOF and lateral resolution in OCT, this article proposes capitalizing on the advantages of SDOCT in a multifocal Bessel beam SDOCT (MBSDOCT) that combines the advantages of both BOCT and MGOCT. The imaging depth of the proposed technique is demonstrated by simulation and experiments. An MBSDOCT system equipped with a superluminescent diode (SLD) operating at a 1301-nm center wavelength (especially apt for depth imaging) is used to image the acinar cells of orange tissue. We obtained a clear imaging depth of $\sim 4 \mathrm{~mm}$, with a sensitivity penalty of $\sim 18.1 \mathrm{~dB}$ at an input power of $9 \mathrm{~mW}$. 

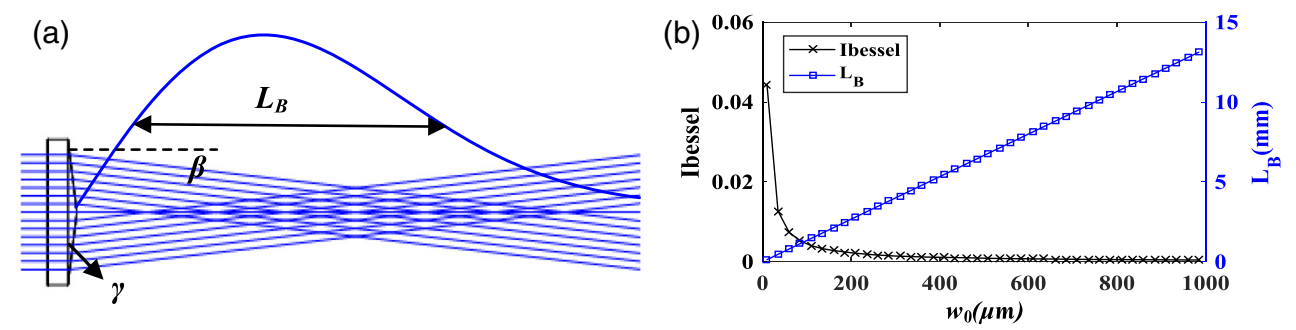

Fig. 1 (a) Schematic diagram of generating Bessel beam and (b) relationships between $I_{\text {bessel }}$, DOF, and $w_{0}$.

\section{Methods}

\subsection{Description of Bessel Beam}

Figure 1(a) shows an axicon with a cone angle $\gamma$ illuminated by a Gaussian beam with $1 / e^{2}$ radius $w_{0}$. Assuming a Gaussian input field with unit power, we may write the intensity distribution behind the axicon as ${ }^{27}$

$I(\tilde{\rho}, \tilde{\mathrm{z}})=\frac{8 \pi}{w_{0}^{2}} N \tilde{\mathrm{z}} \exp \left(-2 \tilde{\mathrm{z}}^{2}\right) J_{0}^{2}(2 \pi N \tilde{\rho})$,

in cylindrical coordinates $\left(\tilde{\rho}=\rho / w_{0}, \tilde{z}=z / L_{B}\right)$, where $N$ is defined as the Fresnel number ${ }^{27}$ and $J_{0}$ is the zero-order Bessel function of the first kind. The axial and radial extents of the beam maximum, generally referred to as the DOF $L_{B}$ and the beam radius $R_{B}$, respectively, are of interest. They can be expressed as ${ }^{28}$

$L_{B}=w_{0} \times\left\{\left[\tan (\beta)^{-1}\right]-\tan (\gamma)\right\}$

and

$R_{B}=\frac{2.4048}{k_{0} \tan \beta} \approx \frac{2.4048}{k_{0} \beta}$,

where $\beta=\arcsin \left(n_{a} \sin \gamma\right)-\gamma, n_{a}$ is the refractive index of the axicon, and $k_{0}$ is the wave number. The peak intensity of the central beam can be expressed as ${ }^{27}$

$I_{\text {bessel }}=I\left(0, \frac{1}{2}\right)=4 \pi \frac{N}{\sqrt{e} w_{0}^{2}}$.
Equation (2) shows that there are two methods of extending the $L_{B}$ : decreasing the $\gamma$ and increasing the $w_{0}$. However, decreasing $\gamma$ will increase $R_{B}$, as shown in Eq. (3). Although increasing $w_{0}$ can increase $L_{B}$ with unchanged $R_{B}$, increasing $w_{0}$ also causes a sharp decline in the peak intensity of the central beam $I_{\text {bessel }}$, as shown in Eq. (4) and Fig. 1(b). Decreased intensity of light incident on the sample will decrease the sensitivity of the system.

\subsection{Experimental Setup}

To benchmark the performance of the proposed MBSDOCT in terms of DOF and SNR, a 1301-nm SDOCT system was built, as shown in Fig. 2(a). The system used an SLD with a 9-mW output power and 85-nm wavelength bandwidth, yielding an axial resolution of $8.8 \mu \mathrm{m}$ in air. A Bessel beam generating system ( $L_{2}, L_{3}$, axicon, $L_{4}$, and $L_{5}$ ) generated the needed Bessel beam. The $\gamma$ of the axicon was $10 \mathrm{deg}$, and the refractive index was 1.43 , resulting in a lateral resolution of $\sim 13 \mu \mathrm{m}$. Interference signals between the reference light and backscattered light, which carry structural information regarding the sample, were collected by a spectrometer with a spectral resolution of $0.05 \mathrm{~nm}$, yielding an imaging depth of $\sim 6 \mathrm{~mm}$. Depth structure information was obtained by inverting the spectral signal. For example, Fig. 2(b) shows the spectral signals from two reflectors at depths of 200 and $400 \mu \mathrm{m}$, respectively, and the structural signal inverted from the spectral signals.

The proposed MBSDOCT is similar to conventional BSDOCT, except that multiple channels are used. Because biological samples move (especially live samples), the samples can easily become displaced. Therefore, the scans were achieved by
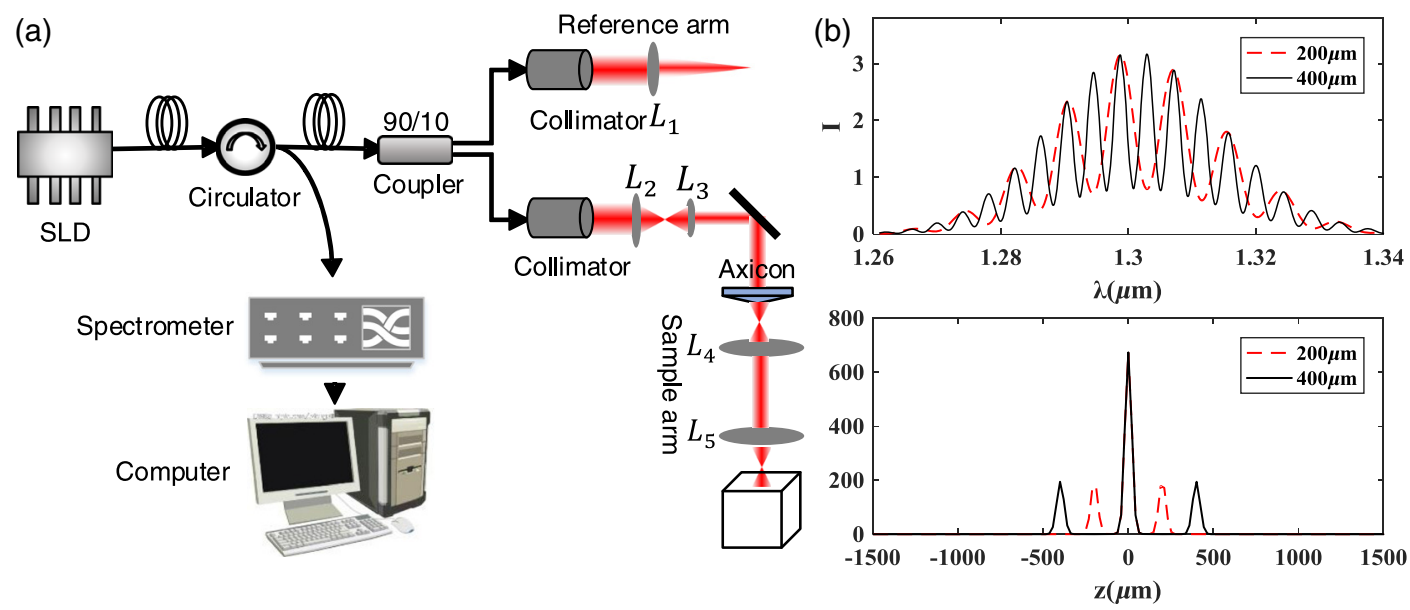

Fig. 2 (a) Bessel beam SDOCT imaging system; (b) top, spectral signals from two reflectors, and bottom, structural signal inverted from the spectral signals. 
shifting axicons, $L_{4}$ and $L_{5}$, simultaneously to change the focus of the beam. It needs to be noted that the axicons, $L_{4}$ and $L_{5}$, were united together in the experimental setup.

The sensitivity of the proposed SDOCT configuration was measured. For Gaussian beam imaging using a conventional lens, the peak sensitivity has conventionally been measured at the focal plane of the lens $\left(L_{5}\right)$, where a mirror was used in place of a biological sample; ${ }^{19}$ an $\sim 101-\mathrm{dB}$ peak sensitivity was measured with an input power of $9 \mathrm{~mW}$. This measurement was made with a difference of $1.6 \mathrm{~mm}$ in the optical path lengths between the sample mirror and the reference mirror. The sensitivity of the Bessel beam setup $\left(N=9.9, w_{0}=170 \mu \mathrm{m}\right)$ was found to be $\sim 83 \mathrm{~dB}$ based on a comparison with the SNR of the Gaussian beam SDOCT (GSDOCT) setup using the same system parameters and sample. $^{27}$

\section{Simulation Results}

\subsection{Comparative Analysis of Different Categories of SDOCT}

In Sec. 1, we introduced several categories of OCT with extended DOF, and this classification method is also applicable

Table 1 Imaging parameters of GSDOCT, ISAM, and BSDOCT.

\begin{tabular}{|c|c|c|}
\hline Method & Theoretical DOF $(\mu \mathrm{m})$ & $\begin{array}{l}\text { Theoretical D } \\
(\mu \mathrm{m})\end{array}$ \\
\hline GSDOCT & $\frac{\pi D^{2}}{2 \lambda_{0}}=204, \lambda_{0}=1.3 \mu \mathrm{m}$ & $\begin{array}{l}2 \frac{\pi}{k_{0} N A}=13 \\
\mathrm{NA}=0.1\end{array}$ \\
\hline ISAM & $\frac{\pi D^{2}}{2 \lambda_{0}} \sqrt{\left(\frac{\mathrm{SNR}_{0}}{\mathrm{SNR}_{L}}\right)^{2}-1}$ & $\begin{array}{c}2 \frac{\pi}{k_{0} N A}=13 \\
N A=0.1\end{array}$ \\
\hline BSDOCT & $\begin{array}{l}w_{0} \times\left\{\left[\tan (\beta)^{-1}\right]-\tan (\gamma)\right\}=1599 \\
w_{0}=120 \mu \mathrm{m}, \gamma=10 \mathrm{deg}\end{array}$ & $2 \times \frac{2.4048}{k_{0} \tan \beta} \approx 13$ \\
\hline
\end{tabular}

to SDOCT. Numerical simulations of methods in each of these categories are useful for providing a theoretical corroboration of the method proposed in this paper. Table 1 describes the parameters of the GSDOCT, ISAM, and BSDOCT, and $D$ is the spot size of Gaussian and Bessel beam, which is considered the lateral resolution here. The spectral resolution was $0.1 \mathrm{~nm}$, and the bandwidth was $80 \mathrm{~nm}$. The BSDOCT simulation in this paper followed published methods for the GSDOCT and ISAM, ${ }^{16}$ except that the Bessel beam replaced the Gauss beam. The numerical simulation consists of the forward model and the reconstruction. The forward model is the interaction between the sample and the incident light, and the spectral datum is collected in this process. The susceptibility of the sample is obtained by inverse Fourier transform of the spectral datum in the reconstruction process.

Figures 3(a), 3(b), and 3(c) show simulation results for the imaging of isotropic point scatterers with diameters of $2 \mu \mathrm{m}$ located randomly, from which we can see that the BSDOCT extended the DOF over those of the two other methods, all with the same lateral resolution.

To illustrate quantitatively the advantages of MBSDOCT in terms of DOF, the relationships between the lateral resolution and the DOF in the methods mentioned in Sec. 1 were analyzed. The DOF of multifocal GSDOCT (MGSDOCT) can be approximated as the sum of the DOFs of all channels, which can be expressed as

$\mathrm{DOF}_{G}=n_{c} \frac{2 \pi n w_{0}^{2}}{\lambda}$,

where $n_{c}$ is the number of channels, $n$ is the refractive index of the tissue sample, and $w_{0}$ is the waist radius of the Gaussian beam. The relationships between the lateral resolution and DOF of GSDOCT and MGSDOCT $\left(n_{c}=2\right)$ are plotted by the black and brown curves as shown in Fig. 4, respectively. ISAM suffers from an SNR that decreases with $1 / z$, which limits its effective DOF, expressed as ${ }^{17}$
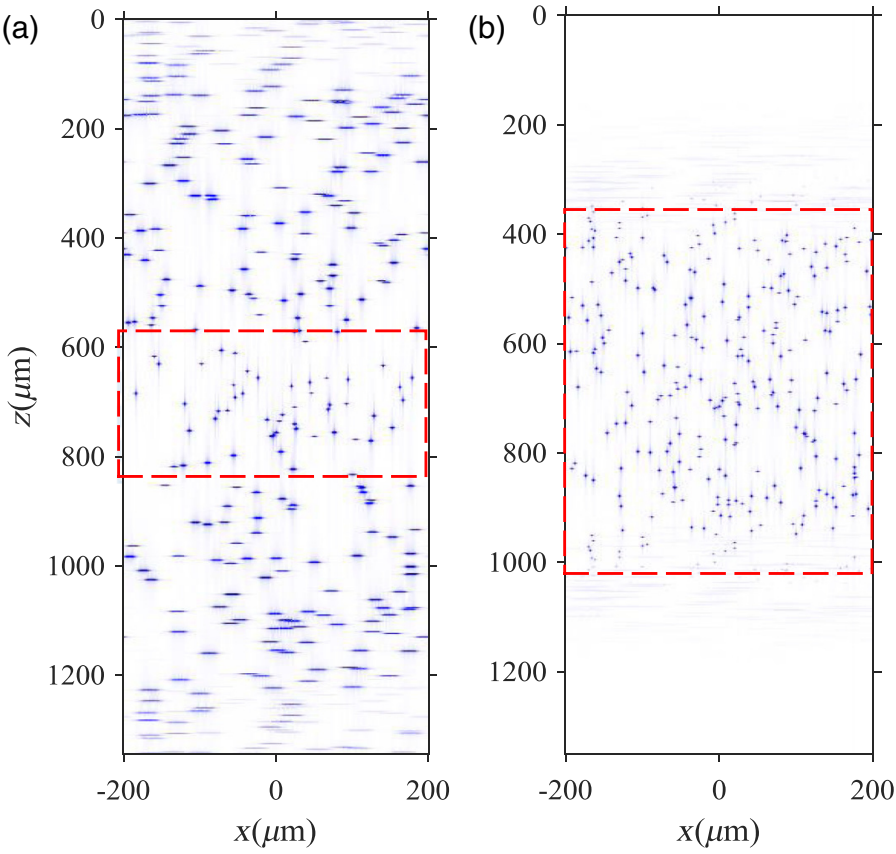

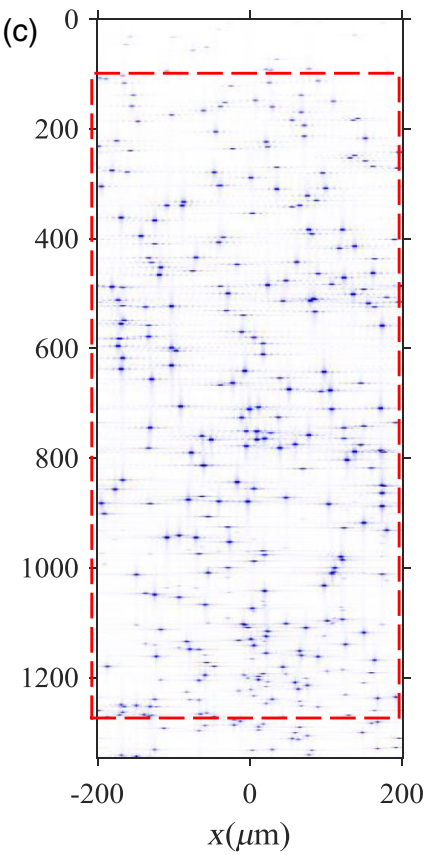

Fig. 3 Simulations of (a) GSDOCT, (b) ISAM, and (c) BSDOCT applied to a scattering particles sample. 


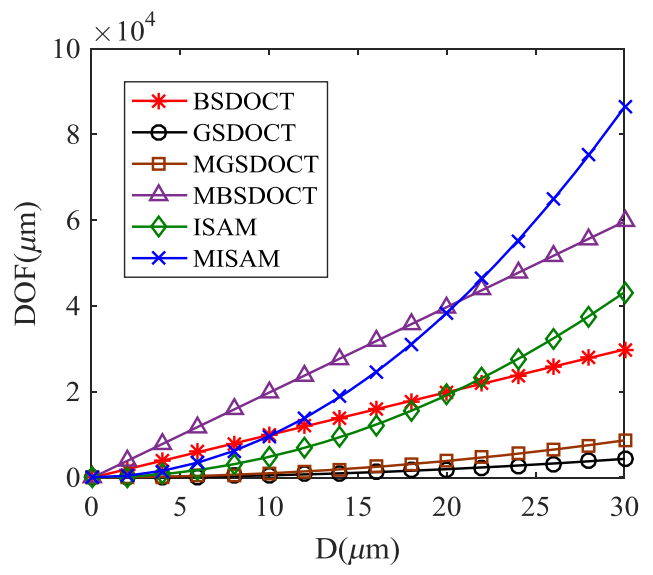

Fig. 4 Relationships between lateral resolution and DOF of GSDOCT, MGSDOCT, ISAM, MISAM, BSDOCT, and MBSDOCT.

$\mathrm{DOF}_{I}=\frac{2 \pi n w_{0}^{2}}{\lambda_{0}} \sqrt{\left(\frac{\mathrm{SNR}_{0}}{\mathrm{SNR}_{L}}\right)^{2}-1}$,

where $\mathrm{SNR}_{0}$ is the $\mathrm{SNR}$ at the focal plane and $\mathrm{SNR}_{L}$ is the lowest tolerable SNR. Similarly, the DOF of MISAM can be approximated as the sum of the DOFs of all channels. The relationships between the lateral resolution and DOF of ISAM and MISAM are plotted by the green and blue curves as shown in Fig. 4, respectively, where $\mathrm{SNR}_{0}=10 \mathrm{SNR}_{L}$ and $n_{c}=2$. In Sec. 2.1, we explained that in BSDOCT, when $\gamma$ is decreased or $w_{0}$ is increased to extend the DOF, either the lateral resolution or the SNR worsens. Therefore, this paper proposes MBSDOCT, which can extend the imaging depth under an unchanged lateral resolution and high SNR. The relationships between the lateral resolution and the DOF of BSDOCT and MBSDOCT are plotted by the pink and red curves as shown in Fig. 4, respectively, where $w_{0}=500 \mu \mathrm{m}$ and $n_{c}=2$.
Figure 4 shows that MISAM and MBSDOCT have prominent advantages in extending the DOF and MBSDOCT has a larger DOF in the range of high lateral resolution (small $D$ ).

\subsection{MBSDOCT Simulation}

Figure 5 shows the results of a computer simulation to verify the feasibility of MBSDOCT. The sample used in the simulation consisted of a series of point scatterers located randomly. The system parameters were consistent with the experimental system introduced in Sec. 2.2.

Figures 5(a) and 5(b) show the BSDOCT images acquired with focal planes at different depths of 0 and $2.2 \mathrm{~mm}$, in which the $w_{0}$ was $170 \mu \mathrm{m}$, yielding a theoretical DOF of $2.2 \mathrm{~mm}$. Figure 5(c) shows the stitched MBSDOCT image synthesized from the data in Figs. 5(a) and 5(b), the depth of which is $\sim 4.4 \mathrm{~mm}$, equivalent to the BSDOCT case, where the $w_{0}$ was $340 \mu \mathrm{m}$, shown in Fig. 5(d). Although Figs. 5(c) and 5(d) present results from the same imaging depth and lateral resolutions, Fig. 5(c) shows a larger signal intensity than Fig. 5(d), as shown in Fig. 5(e). We deduced that the SNR of MBSDOCT would be higher in the actual experiment, which was later verified.

Based on these analyses, the MBSDOCT image with a smaller $w_{0}$ and more channels was predicted to have a higher SNR than the MBSDOCT image with a larger $w_{0}$ and fewer channels, which was later verified experimentally.

\section{Experimental Results}

Two datasets were acquired separately using a BSDOCT setup. The focal planes of the scanning beams were adjusted before each scan. As shown in Fig. 6, two datasets (a) and (b) were acquired with focal planes at 0 and $1.5 \mathrm{~mm}$, respectively. Figure 6(c) shows the MBSDOCT image of the two datasets. The SDOCT system had a $w_{0}$ of $207 \mu \mathrm{m}$, yielding a DOF of $2.7 \mathrm{~mm}$ in air. The sample was orange tissue, and we assumed (a) channel1

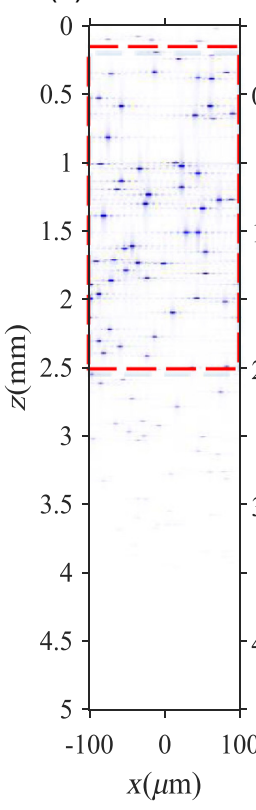

(b) channel2

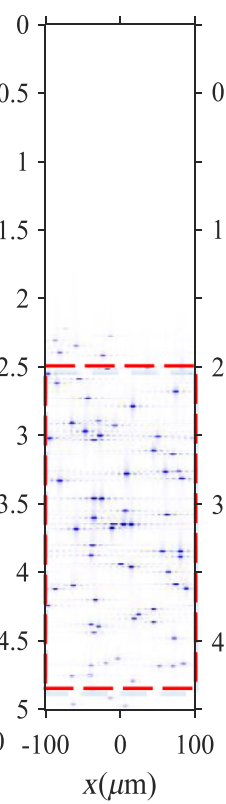

(c) MBSDOCT

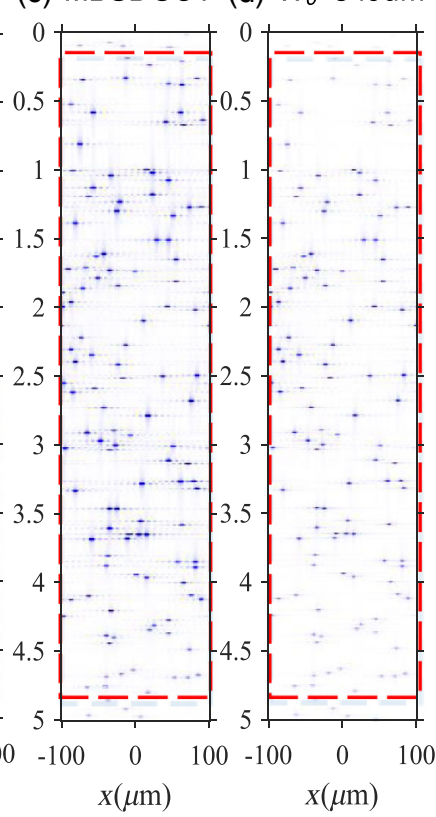

(e)

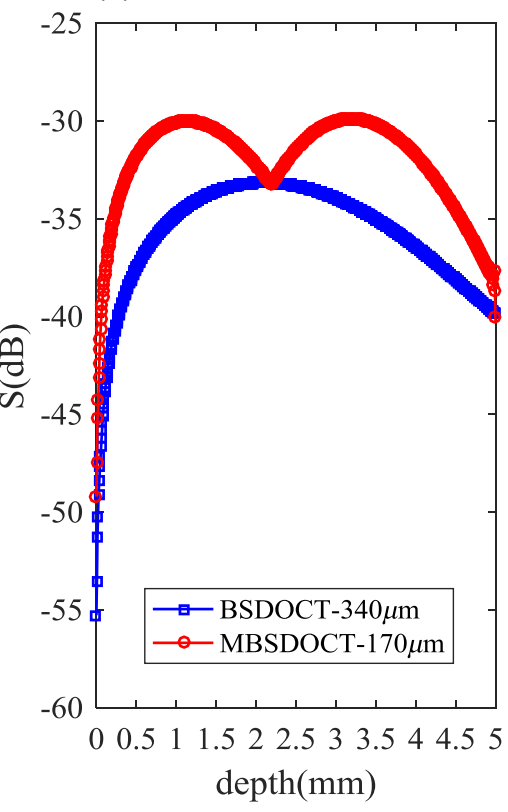

Fig. 5 (a)-(d) Simulation results of MBSDOCT $\left(w_{0}=170 \mu \mathrm{m}\right)$ and BSDOCT $\left(w_{0}=340 \mu \mathrm{m}\right)$ and (e) signal intensity $(\mathrm{dB})$ of (c) and (d). 
(a) channel 1

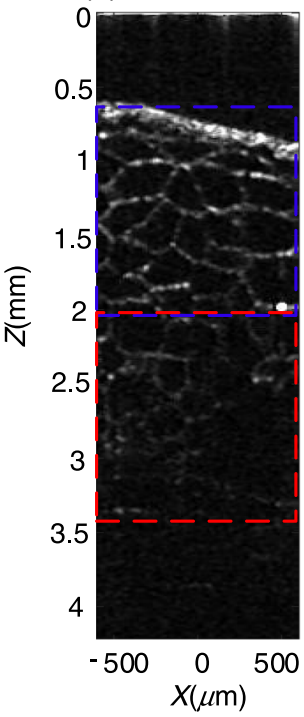

(b) channel 2

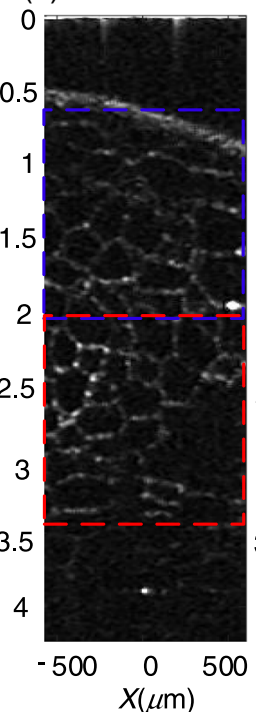

(c) MBSDOCT

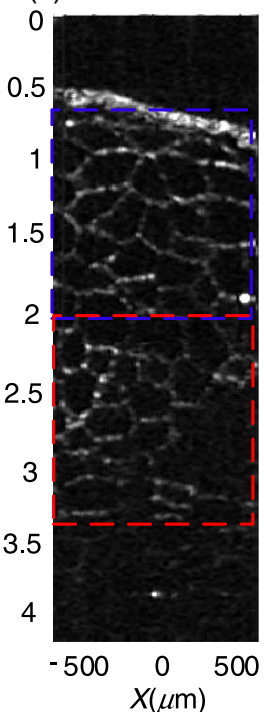

(d)

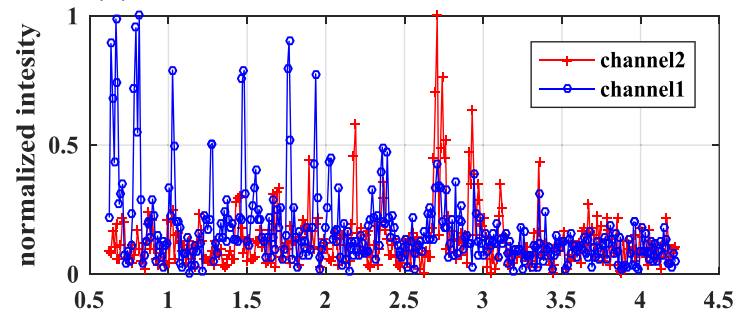

(e)

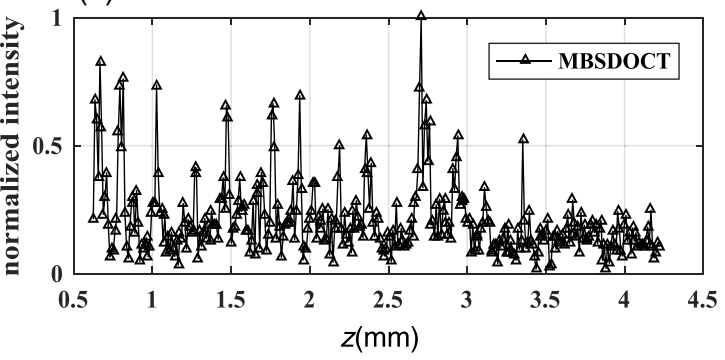

Fig. 6 (a)-(c) Two channels of BSDOCT images and the resulting MBSDOCT image, respectively; (d) signal intensity of one transverse point selected from the two BSDOCT figures; and (e) signal intensity of one transverse point selected from the MBSDOCT image.

a refractive index of 1.33 for water. The image from each channel becomes unclear far away from the focal plane. Figure 6(a) shows an estimated effective DOF of $\sim 1.5 \mathrm{~mm}$ (from the blue dotted frame); Fig. 6(b) shows an estimated effective DOF of $\sim 1.5 \mathrm{~mm}$ (from the red dotted frame); and Fig. 6(c) shows an estimated effective DOF of $\sim 3 \mathrm{~mm}, \sim 2$ times greater than either single channel. Figures 6(d) and 6(e) indicate that the image obtained by MBSDOCT exhibited high signal intensity throughout the entire depth.

Table 2 shows the SNRs of the images outlined by the blue and red dashed boxes in the three graphs in Fig. 6. Because there is a transverse offset between the two BSDOCT images, the registration image is not the sum of the two images; rather, the registration image is spliced by the clear part of the two BSDOCT images. The table shows that the MBSDOCT image exhibited a high SNR throughout the entire depth.

The cell structure size of the two channel images in Fig. 6 is inconsistent. There may be two reasons for this problem. First, the measuring arm had a lateral swing while moving in the longitudinal direction, and the lengthened optical path difference resulting from this swing, considered the vertical depth of the structure, inevitably causes the different sizes of cells imaged in the two channels, both in the longitudinal and lateral directions. Second, the scan speed of the spectrometer used in this experiment was slow, and the imaging of one channel takes several minutes, during which the orange tissue changes.

Table 2 SNRs of the three images in Fig. 6.

\begin{tabular}{|c|c|c|}
\hline Image & SNR1 $(0.5$ to $2 \mathrm{~mm})$ & SNR2 (2 to $3.5 \mathrm{~mm})$ \\
\hline Channel 1 & 20.6 & 17 \\
\hline Channel 2 & 16.4 & 20.1 \\
\hline MBSDOCT & 20.6 & 20.1 \\
\hline
\end{tabular}

Therefore, a stable mobile station, precise adjustment of the optical system, and a fast spectrometer are needed to avoid inconsistent cell sizes in different channels images.

In Fig. 7, three datasets (a), (b), and (c) were acquired with focal planes at $0,1.5$, and $3 \mathrm{~mm}$, respectively. Figure 7(d) shows the MBSDOCT image of the three datasets, with an effective DOF of $\sim 4 \mathrm{~mm}$. The SDOCT system had a $w_{0}$ of $170 \mu \mathrm{m}$, yielding a 2.2-mm DOF in air. From Figs. 7(e) and 7(f), we can see that the MBSDOCT image exhibited a high SNR throughout the entire depth. The SNR in the red dashed frame of Fig. 7(a) was 22.1, which was higher than that shown in Fig. 6(a). Therefore, the MBSDOCT image with a smaller $w_{0}$ and more channels exhibited a higher SNR than the MBSDOCT image with a larger $w_{0}$ and fewer channels. However, when the number of channels increases, the system becomes more complex and the acquisition time becomes longer.

In Sec. 3.2, we demonstrated by simulation that increasing $w_{0}$ can also extend the DOF. However, the image taken with $w_{0}=340 \mu \mathrm{m}$ exhibited lower signal intensity compared with the MBSDOCT image taken with $w_{0}=170 \mu \mathrm{m}$ when their DOFs were the same. Experiments with the same parameters as were used in the simulation were implemented to demonstrate the predicted result; however, the focal plane of each channel was shifted by $1 \mathrm{~mm}$.

Figures 8(a) and 8(b) show the BSDOCT images with a $w_{0}$ of $170 \mu \mathrm{m}$, and Fig. 8(c) shows the MBSDOCT image resulting from these two datasets. Figure 8(d) shows the BSDOCT image with a $w_{0}$ of $340 \mu \mathrm{m}$. Figures 8(c) and 8(d) show approximately the same image depth; however, the MBSDOCT image exhibited a larger signal intensity, as shown in Fig. 8(e). Similarly, the SNRs of the images outlined by the blue dashed boxes in the two pictures were calculated. The results indicate that the image shown in Fig. 8(d) suffered from a lower SNR of 20.33, whereas the MBSDOCT image exhibited a larger SNR of 22.09. Therefore, the MBSDOCT setup with $w_{0}$ exhibited a larger SNR than the BSDOCT setup with $2 w_{0}$, although both setups had the same lateral resolution and theoretical DOF. 
(a)

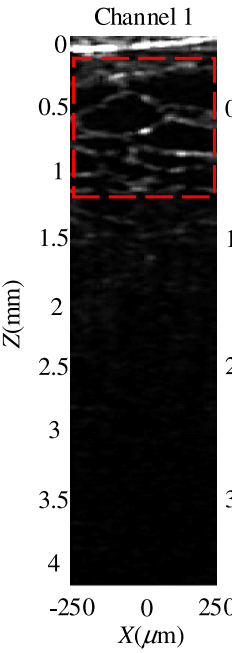

(b)

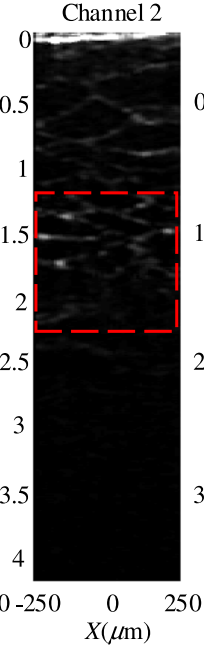

(c)

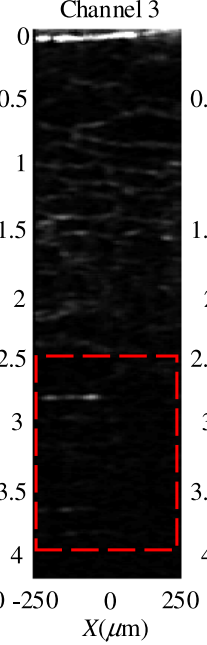

(d)

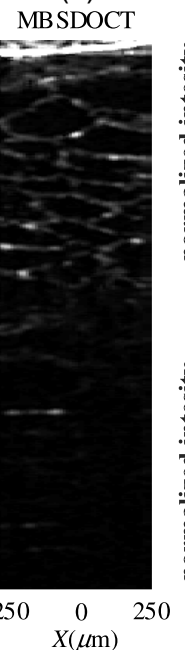

(e)
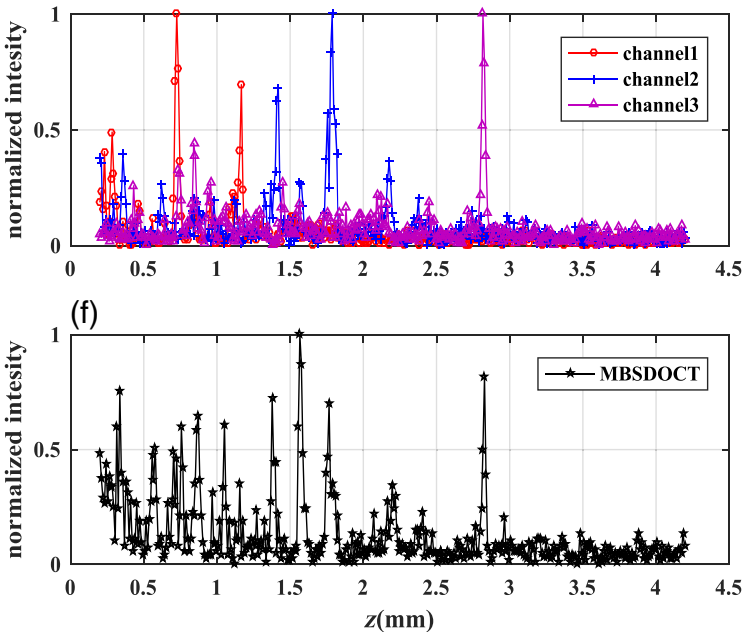

Fig. 7 (a)-(d) Three channels of BSDOCT images and the resulting MBSDOCT image, respectively; (e) signal intensity of one transverse point selected from the three BSDOCT figures; and (f) signal intensity of one transverse point selected from the MBSDOCT image.
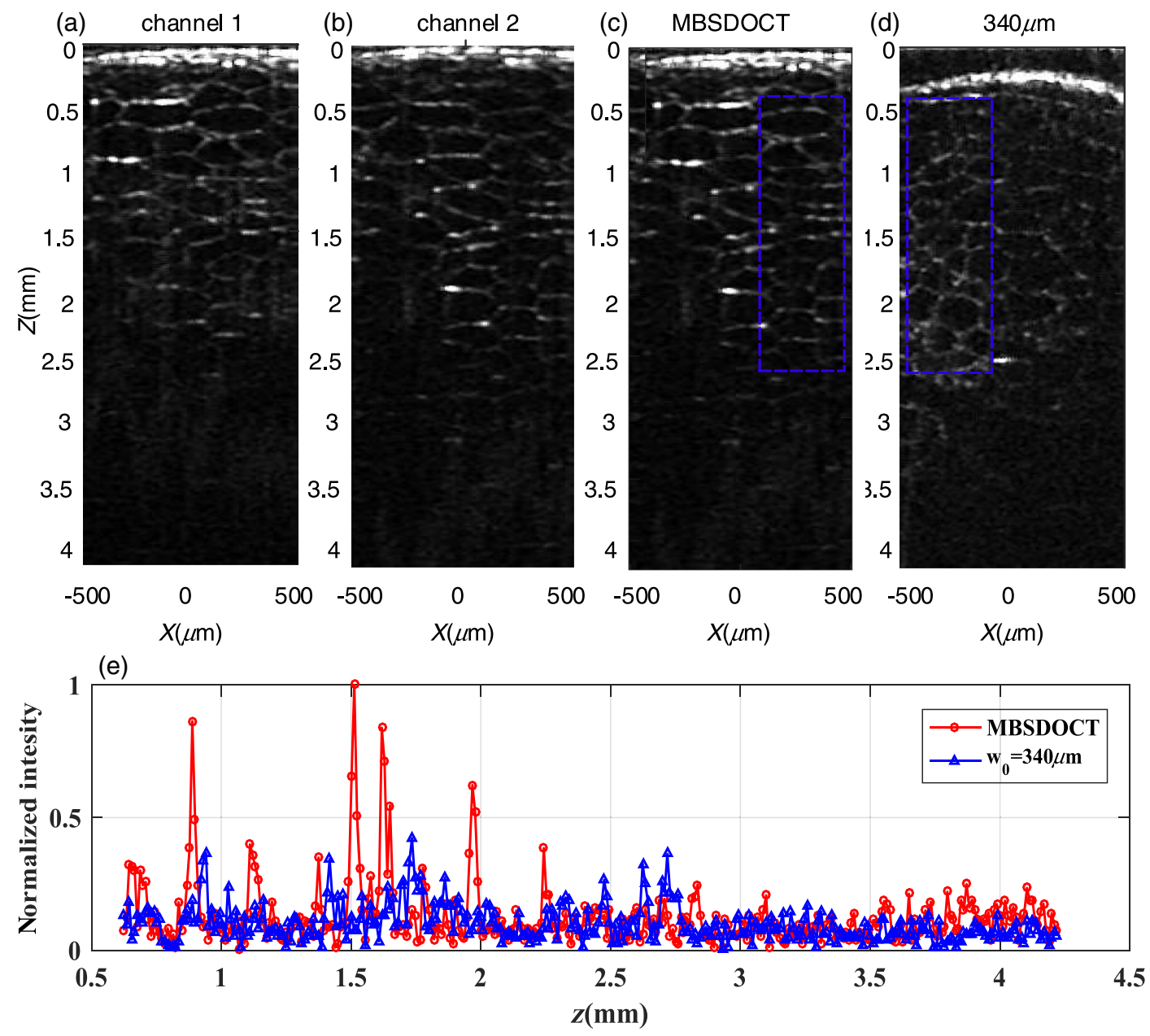

Fig. 8 (a)-(c) Two channels of BSDOCT images and the resulting MBSDOCT image with $w_{0}$ of $170 \mu \mathrm{m}$; (d) BSDOCT image with $w_{0}$ of $340 \mu \mathrm{m}$; and (e) signal intensity of one transverse point selected from the two figures. 
Table 3 Comparison of BSDOCT with different $w_{0}$.

\begin{tabular}{lccc}
\hline & Bessel 1 & Bessel 2 & Bessel 3 \\
\hline Wrist radius $w_{0}(\mu \mathrm{m})$ & 170 & 207 & 340 \\
Fresnel number $N$ & 9.9 & 12 & 19.8 \\
Theoretical DOF $(\mathrm{mm})$ & 2.2 & 2.7 & 4.5 \\
Measured DOF $(\mathrm{mm})$ & 2 & 2.5 & 3 \\
Theoretical SNR penalty $(\mathrm{dB})$ & 18.2 & 19.9 & 24 \\
Measured SNR penalty $(\mathrm{dB})$ & 18.1 & 21.9 & 29.8 \\
\hline
\end{tabular}

Table 3 gives the results of different BSDOCT experimental sets under different $w_{0}$. These results are consistent with the theory: the larger a $w_{0}$ the system has, the lower its SNR. The theoretical SNR penalty relative to GSDOCT can be expressed as a function of the Fresnel number $N^{27}$

$\operatorname{SNR}$ penalty $(\mathrm{dB})=20 \log \left(\frac{2}{\sqrt{e} N}\right)=20 \log \left(\frac{1.213}{N}\right)$.

The reason for which the imaging depth does not reach the theoretical value and for which the number of channels cannot be further increased is the lower power of the light source. Larger input power has greater imaging depth and SNR; however, the biological tissue's damage threshold must be considered.

\section{Conclusion}

In this paper, we demonstrated that the proposed MBSDOCT can improve imaging depth and SNR. The simulation results showed that the MBSDOCT can obtain a larger DOF and signal intensity, and these results were verified by experiments on acinar cells of orange tissue. When three foci were used, we obtained an imaging depth of $\sim 4 \mathrm{~mm}$ with a lateral resolution of $\sim 13 \mu \mathrm{m}$, and this imaging depth can increase when a larger input power is used. Furthermore, MBSDOCT images with a smaller $w_{0}$ and more channels were demonstrated to achieve a higher SNR than MBSDOCT images with a larger $w_{0}$ and fewer channels, although they have the same imaging depth and lateral resolution. Therefore, the proposed MBSDOCT method is offers an important advance for the deep imaging of biological tissue and diagnosis of deep lesions in medicine. In addition, the multifocal Bessel beam scheme is suitable for a variety of OCT modes, compared with SDOCT.

\section{Disclosures}

The authors have no relevant financial interests in the article and no other potential conflicts of interest to disclose.

\section{Acknowledgments}

This research was funded by the State Key Lab Precision Measurement Technology and Instruments, Tsinghua University, and the National Major Scientific Instrument and Equipment Development Project of China (Grant Nos. 2012YQ200182 and 2012YQ0901670602).

\section{References}

1. D. Huang et al., "Optical coherence tomography," Science 254(5035), 1178-1181 (1991).

2. A. F. Fercher et al., "Optical coherence tomography-principles and applications," Rep. Prog. Phys. 66(2), 239-303 (2003).

3. G. J. Tearney et al., "In vivo endoscopic optical biopsy with optical coherence tomography," Science 276(5321), 2037-2039 (1997).

4. X. Y. Zhang et al., "Dual-band spectral-domain optical coherence tomography for in vivo imaging the spectral contrasts of the retinal nerve fiber layer," Opt. Express 19(20), 19653-19667 (2011).

5. Y. Zhao et al., "Evaluation of burn severity in vivo in a mouse model using spectroscopic optical coherence tomography," Biomed. Opt. Express 6(9), 3339-3345 (2015).

6. M. R. Hee et al., "Optical coherence tomography of the human retina," Arch. Ophthalmol. 113(3), 325 (1995).

7. M. R. Hee et al., "Quantitative assessment of macular edema with optical coherence tomography," Arch. Ophthalmol. 113(8), 1019 (1995).

8. Y. K. K. Tao et al., "Intraoperative spectral domain optical coherence tomography for vitreoretinal surgery," Opt. Lett. 35(20), 3315-3317 (2010).

9. S. P. Chong et al., "Quantitative microvascular hemoglobin mapping using visible light spectroscopic optical coherence tomography," Biomed. Opt. Express 6(4), 1429-1450 (2015).

10. J. Holmes et al., "Multi-channel Fourier domain OCT system with superior lateral resolution for biomedical applications," Proc. SPIE 6847, 684700 (2008).

11. J. Holmes, "Theory and applications of multi-beam OCT," Proc. SPIE 7139, 713908 (2008).

12. Y. Xu et al., "Automated interferometric synthetic aperture microscopy and computational adaptive optics for improved optical coherence tomography," Appl. Opt. 55(8), 2034-2041 (2016).

13. T. S. Ralston et al., "Interferometric synthetic aperture microscopy," Nat. Phys. 3(2), 129-134 (2007).

14. P. S. Carney et al., "Automation of interferometric synthetic aperture microscopy," in Frontiers in Optics, FTu3D.3 (2015).

15. B. J. Davis et al., "Interferometric synthetic aperture microscopy: computed imaging for scanned coherent microscopy," Sens. Basel 8(6), 3903-3931 (2008).

16. B. J. Davis et al., "Nonparaxial vector-field modeling of optical coherence tomography and interferometric synthetic aperture microscopy," J. Opt. Soc. Am. A 24(9), 2527-2542 (2007).

17. Y. Xu et al., "Multifocal interferometric synthetic aperture microscopy," Opt. Express 22(13), 16606-16618 (2014).

18. C. Blatter et al., "High-speed functional OCT with self-reconstructive Bessel illumination at 1300 nm," Proc. SPIE 8091, 809104 (2011).

19. K. S. Lee and L. P. Rolland, "Bessel beam spectral-domain highresolution optical coherence tomography with micro-optic axicon providing extended focusing range," Opt. Lett. 33(15), 1696-1698 (2008).

20. R. A. Leitgeb et al., "Extended focus depth for Fourier domain optical coherence microscopy," Opt. Lett. 31(16), 2450-2452 (2006).

21. A. Curatolo et al., "Quantifying the influence of Bessel beams on image quality in optical coherence tomography," Sci. Rep. 6, 23483 (2016).

22. J. F. de Boer et al., "Improved signal-to-noise ratio in spectral-domain compared with time-domain optical coherence tomography," Opt. Lett. 28(21), 2067-2069 (2003).

23. M. Wojtkowski et al., "Full range complex spectral optical coherence tomography technique in eye imaging," Opt. Lett. 27(16), 1415-1417 (2002).

24. M. Wojtkowski et al., "Real-time in vivo imaging by high-speed spectral optical coherence tomography," Opt. Lett. 28(19), 1745-1747 (2003).

25. Z. H. Hu et al., "Automated segmentation of neural canal opening and optic cup in 3D spectral optical coherence tomography volumes of the optic nerve head," Invest. Ophthalmol. Visual Sci. 51(11), 5708-5717 (2010).

26. B. J. Kaluzny, "Spectral optical coherence tomography: a novel technique for cornea imaging," Cornea 25(8), 960 (2006).

27. D. Lorenser et al., "Energy-efficient low-Fresnel-number Bessel beams and their application in optical coherence tomography," Opt. Lett. 39(3), 548-551 (2014).

28. N. Weber et al., "Highly compact imaging using Bessel beams generated by ultraminiaturized multi-micro-axicon systems," J. Opt. Soc. Am. A 29(5), 808-816 (2012). 
Luying $\mathrm{Yi}$ is a PhD student in optical engineering at the Department of Precision Instruments, Tsinghua University. She received her bachelor's degree in optoelectronics and information engineering at Tianjin University in 2015. She is currently studying the spectral-domain optical coherence tomography under the guidance of Professor Sun in State Key Laboratory of Precision Measurement Technology \& Instruments. Her current works include the brain imaging and deep imaging.

Liqun Sun received his BS degree in applied optics from Beijing Institute of Technology in 1988, and his PhD in physical electronics in 2000 from Xi'an Jiao Tong University, China. He had been an Engineer in Xi'an Institute of Applied Optics since 1988. In 2000, he joined the Department of Precision Instruments, Tsinghua university. He is now a professor of optical engineering. His research interests include spectrum measurement technology, optical sensing and bio-optics.

Wuwen Ding is a PhD student in optical engineering at the Department of Precision Instruments, Tsinghua University. He received his bachelor's degree in Measurement and Control Technology and Instrumentation Program at Tsinghua University in 2012. His works focus on gas absorption spectroscopy. He currently studying tunable diode laser absorption spectroscopy (TDLAS) and chirped laser dispersion spectroscopy (CLADS) under the guidance of Professor Sun in State Key Laboratory of Precision Measurement Technology \& Instruments. 